\title{
Disk and wind interaction in the young stellar object MWC 297 spatially resolved with AMBER/VLTI ${ }^{\star}$
}

\author{
F. Malbet ${ }^{1}$, M. Benisty ${ }^{1}$, W.-J. de Wit ${ }^{1}$, S. Kraus ${ }^{2}$, A. Meilland ${ }^{3}$, F. Millour ${ }^{1,4}$, E. Tatulli ${ }^{1,5}$, J.-P. Berger ${ }^{1}$, \\ O. Chesneau ${ }^{3}$, K.-H. Hofmann 2 , A. Isella ${ }^{5,13}$, A. Natta ${ }^{5}$, R.G. Petrov ${ }^{4}$, T. Preibisch ${ }^{2}$, P. Stee ${ }^{3}$, L. Testi ${ }^{5}$, G. Weigelt ${ }^{2}$, \\ P. Antonelli ${ }^{3}$, U. Beckmann ${ }^{2}$, Y. Bresson ${ }^{3}$, A. Chelli ${ }^{1}$, M. Dugué ${ }^{3}$, G. Duvert ${ }^{1}$, S. Gennari ${ }^{5}$, L. Glück ${ }^{1}$, P. Kern ${ }^{1}$, \\ S. Lagarde ${ }^{3}$, E. Le Coarer ${ }^{1}$, F. Lisi ${ }^{5}$, K. Perraut ${ }^{1}$, P. Puget ${ }^{1}$, F. Rantakyrö 6 , S. Robbe-Dubois ${ }^{4}$, A. Roussel ${ }^{3}$, G. Zins ${ }^{1}$, \\ M. Accardo ${ }^{5}$, B. Acke ${ }^{1,14}$, K. Agabi ${ }^{4}$, E. Altariba ${ }^{1}$, B. Arezki ${ }^{1}$, E. Aristidi ${ }^{4}$, C. Baffa ${ }^{5}$, J. Behrend ${ }^{2}$, T. Blöcker ${ }^{2}$, \\ S. Bonhomme ${ }^{3}$, S. Busoni ${ }^{5}$, F. Cassaing ${ }^{7}$, J.-M. Clausse ${ }^{3}$, J. Colin ${ }^{3}$, C. Connot ${ }^{2}$, A. Delboulbé ${ }^{1}$, A. Domiciano de \\ Souza $^{4,3}$, T. Driebe ${ }^{2}$, P. Feautrier ${ }^{1}$, D. Ferruzzi ${ }^{5}$, T. Forveille ${ }^{1}$, E. Fossat ${ }^{4}$, R. Foy $^{8}$, D. Fraix-Burnet ${ }^{1}$, A. Gallardo ${ }^{1}$, \\ E. Giani ${ }^{5}$, C. Gil ${ }^{1,15}$, A. Glentzlin ${ }^{3}$, M. Heiden ${ }^{2}$, M. Heininger ${ }^{2}$, O. Hernandez Utrera ${ }^{1}$, D. Kamm ${ }^{3}$, M. Kiekebusch ${ }^{6}$, \\ D. Le Contel ${ }^{3}$, J.-M. Le Contel ${ }^{3}$, T. Lesourd ${ }^{9}$, B. Lopez ${ }^{3}$, M. Lopez ${ }^{9}$, Y. Magnard ${ }^{1}$, A. Marconi ${ }^{5}$, G. Mars ${ }^{3}$, \\ G. Martinot-Lagarde ${ }^{9,3}$, P. Mathias ${ }^{3}$, P. Mège ${ }^{1}$, J.-L. Monin ${ }^{1}$, D. Mouillet ${ }^{1,16}$, D. Mourard ${ }^{3}$, E. Nussbaum ${ }^{2}$,

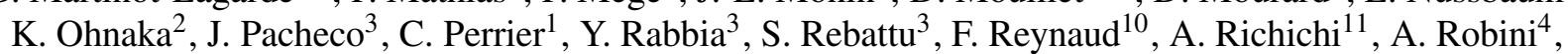 \\ M. Sacchettini ${ }^{1}$, D. Schertl ${ }^{2}$, M. Schöller ${ }^{6}$, W. Solscheid ${ }^{2}$, A. Spang ${ }^{3}$, P. Stefanini ${ }^{5}$, M. Tallon ${ }^{8}$, I. Tallon-Bosc ${ }^{8}$, \\ D. Tasso ${ }^{3}$, F. Vakili ${ }^{4}$, O. von der Lühe ${ }^{12}$, J.-C. Valtier ${ }^{3}$, M. Vannier ${ }^{4,6,17}$, and N. Ventura ${ }^{1}$
}

(Affiliations can be found after the references)

Received 26 July 2005/ Accepted 11 October 2005

\begin{abstract}
The young stellar object MWC 297 is an embedded B1.5Ve star exhibiting strong hydrogen emission lines and a strong near-infrared continuum excess. This object has been observed with the VLT interferometer equipped with the AMBER instrument during its first commissioning run. AMBER/VLTI is currently the only near infrared interferometer that can observe spectrally dispersed visibilities. MWC 297 has been spatially resolved in the continuum with a visibility of $0.50_{-0.10}^{+0.08}$ as well as in the $\mathrm{Br} \gamma$ emission line where the visibility decreases to $0.33 \pm 0.06$. This change in the visibility with wavelength can be interpreted by the presence of an optically thick disk responsible for the visibility in the continuum and of a stellar wind traced by the $\mathrm{Br} \gamma$ emission line and whose apparent size is $40 \%$ larger. We validate this interpretation by building a model of the stellar environment that combines a geometrically thin, optically thick accretion disk model consisting of gas and dust, and a latitude-dependent stellar wind outflowing above the disk surface. The continuum emission and visibilities obtained from this model are fully consistent with the interferometric AMBER data. They agree also with existing optical, near-infrared spectra and other broad-band near-infrared interferometric visibilities. We also reproduce the shape of the visibilities in the Br $\gamma$ line as well as the profile of this line obtained at an higher spectral resolution with the VLT/ISAAC spectrograph, and those of the $\mathrm{H} \alpha$ and $\mathrm{H} \beta$ lines. The disk and wind models yield a consistent inclination of the system of approximately $20^{\circ}$. A picture emerges in which MWC 297 is surrounded by an equatorial flat disk that is possibly still accreting and an outflowing wind that has a much higher velocity in the polar region than at the equator. The AMBER/VLTI unique capability of measuring spectral visibilities therefore allows us for the first time to compare the apparent geometry of a wind with the disk structure in a young stellar system.
\end{abstract}

Key words. accretion, accretion disks - techniques: interferometric - stars: pre-main sequence - planetary systems: protoplanetary disks stars: emission-line, Be - stars: individual: MWC297

\section{Introduction}

Pre-main sequence stars in the intermediate mass range, called Herbig Ae and Be stars (HAeBe), are observed to be surrounded by circumstellar material which reveals itself by discrete emission lines and by continuous excess emission in the spectral energy distribution (SED). The spatial distribution of this material however has been subject to debate: both geometrically flat disk models and spherically symmetric envelope models can reproduce the observed SED. Using viscous accretion disk models, Hillenbrand et al. (1992) proposed a disk-like geometry for the circumstellar material that however needs a central cavity to fit the near-infrared (NIR) excess. Considerations of the physical

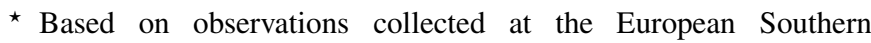
Observatory, Paranal, Chile, within the commissioning programme 60. A-9054 (A). reality of these central cavities (Kenyon et al. 1993) and successful fits to HAeBe SEDs by dust envelopes (Berrilli et al. 1992; di Francesco et al. 1994) or composite envelope-disk models (Miroshnichenko et al. 1999) introduced controversy on the geometry of the circumstellar material.

A break-through occurred with high resolution interferometric observations of HAeBe stars first presented by Millan-Gabet et al. (2001) and Tuthill et al. (2001). These observations revealed that the geometries observed in the near-infrared bands were closer to either ring-like or spherically symmetric rather than disk-like. New models introducing passive, star-irradiated circumstellar disks with puffed up inner rims were found to be consistent with both the interferometric and photometric observations (Natta et al. 2001; Dullemond et al. 2001) although no simultaneous fits were performed. In this scenario the inner part of the circumstellar disk edge puffs up due to direct 
Table 1. AMBER observation log from 31 May 2004 (UT).

\begin{tabular}{lclllll}
\hline \hline Star & $\begin{array}{l}\text { DIT } \\
(\mathrm{ms})\end{array}$ & $\begin{array}{l}\text { UT } \\
\text { start }\end{array}$ & $\begin{array}{l}\text { UT } \\
\text { end }\end{array}$ & $\begin{array}{l}\text { Nb } \\
\text { exp. }\end{array}$ & $\begin{array}{l}u \\
(\mathrm{~m})\end{array}$ & $\begin{array}{l}v \\
(\mathrm{~m})\end{array}$ \\
\hline MWC 297 & 107 & $06: 02$ & $06: 13$ & 18 & 28.19 & 33.57 \\
MWC 297 & 31 & $06: 26$ & $06: 37$ & 40 & 29.93 & 33.37 \\
HD 177756 & 31 & $08: 10$ & $08: 18$ & 30 & 32.92 & 32.85 \\
HD 177756 & 107 & $08: 21$ & $08: 33$ & 14 & 33.20 & 32.69 \\
\hline
\end{tabular}

irradiation by the central star, roughly equivalent to the ringlike structures found with interferometry. The location of this inner rim is near the dust sublimation radius, and the subsequent (dust-free) inner holes are much larger than the ad-hoc inner holes proposed in Hillenbrand et al. (1992). Recent studies show that there seems to exist a correlation between the distance of the inner rim and the luminosity of the central star (Monnier \& Millan-Gabet 2002). This relation holds well for the Herbig Ae and late type Be stars, but breaks down for the most luminous HBe stars (Eisner et al. 2004; Monnier et al. 2005). The latter authors in fact demonstrate that the early-type HBe have inner rims that are too close to the star with respect to the star's luminosity. The HBe seem to better match the "classical" viscous accretion disk model similar to the one initially applied by Hillenbrand et al. (1992). A marked difference however is that now the inner hole should be filled with optically thick gas to effectively shield the dust from destruction at relatively small distances from the inner rim, assuming that dust is what is observed in interferometry. Additional strong evidence for a different disk character near the early-type HBe stars is found in the spectro-polarimetric fingerprint of $\mathrm{H} \alpha$ lines (Vink et al. 2002). These authors prefer to draw the analogy with the geometrically flat gaseous disks present near the classical Be stars.

The geometry of circumstellar material near HAeBe stars thus seems to differ between the early-type and late-type members of the group, which is not surprising given the increasing interaction between star and disk for the early type stars. For the HAe stars a successful working model exists, while on the other hand, the disk structure near the $\mathrm{HBe}$ stars and their intricate star-disk interactions still escape a good understanding. In this study we present high spatial resolution, intermediate spectral resolution interferometric observations of the early-type Herbig Be star MWC 297 using AMBER/VLTI. This enigmatic star appeared in the original HAeBe list compiled by Herbig (1960). The star displays a strong emission line spectrum and the character of the underlying photosphere was revealed in the detailed study by Drew et al. (1997) to be B1.5Ve. Its rather well determined stellar parameters and its high NIR luminosity render this star the perfect target to investigate in detail the geometry of the circumstellar material near the early type HBe stars.

The paper is organized as follows. Section 2 presents the new observations made with AMBER and ISAAC. In Sect. 3, we describe the AMBER data processing to produce a reliable result presented in Sect. 4 for the visibilities both in the continuum and in the Br $\gamma$ line. In Sect. 5 we present an attempt to model the environment of MWC 297 with a disk and a wind. This model and the consequences are discussed in Sect. 6 and summarized in Sect. 7.

\section{Observations}

\subsection{AMBER observations}

MWC 297 was observed on 31 May 2004 during the second night of the first commissioning run of the AMBER instrument on the UT2-UT3 $(47 \mathrm{~m})$ baseline of the Very Large Telescope
Interferometer (VLTI). AMBER is the VLTI beam combiner operating in the near-infrared (Petrov et al. 2007). The instrument is based on spatial filtering with fibers and multiaxial fringe coding (i.e. AMBER combines the beams at an angle that results in fringes modulated in the spatial direction). The interferometric beam is anamorphized perpendicularly to the fringe coding in order to be injected into the slit of a spectrograph. The instrument can operate at spectral resolutions up to 10000 and efficiently deliver spectrally dispersed visibilities.

MWC 297 was measured in the [1980, $2230 \mathrm{~nm}$ ] spectral range in the MR-K spectral mode (spectral resolution of 1500) with 2 elementary detector integration times (DIT) of $31 \mathrm{~ms}$ and $107 \mathrm{~ms}$. Table 1 gives the log of the AMBER observations. HD 177756, whose spectral type is B9V, was used to calibrate the visibilities. Its diameter is $0.60 \pm 0.06$ as computed by the ASPRO searchCalib tool developed at the Jean-Marie Mariotti Center.

The observations of MWC 297 were carried out under specific conditions since it was the first AMBER commissioning run on the VLTI. The bright Bry line of MWC 297 has been originally observed in order to perform a spectral calibration of AMBER. Detailed analysis of the commissioning data from this run and later ones has shown that the optical train of the UT telescopes were affected by non-stationary high-amplitude vibrations. Because of the small number of observations on MWC 297, and because the amplitude of the vibrations might undergo rapid variations, the calibration of our measurements must therefore be regarded with care. In order to investigate the errors on the visibility measurements and check their consistency, we have used different data reduction methods and different data selection schemes which are described below. However the vibrations do not impact the spectral dependence of the visibility since they affect the spectral range as a whole.

\subsection{ISAAC observations}

MWC 297 was observed in service mode on 13 July 2004 with the ESO VLT UT1 telescope under modest seeing conditions $\left(\sim 1.5^{\prime \prime}\right.$ in the visual). The ISAAC near-infrared spectrograph was employed in the short wavelength medium resolution mode with a $0.3^{\prime \prime}$ wide slit. This instrument setup delivered a resolution of $\lambda / \Delta \lambda \sim 8900$ at the $\operatorname{Br} \gamma$ wavelength. The raw data were flat-fielded, wavelength-calibrated and corrected for telluric absorption using standard techniques and observations taken from the ISAAC calibration plan by the ESO staff astronomers. A detailed account of the observations and the data reduction is given in García Lopez et al. (2006).

\subsection{Existing photometric and interferometric data sets}

Extensive photometric and interferometric data exist for MWC 297. Broad-band photometric data were collected and presented by Pezzuto et al. (1997). This data set consists of UBVRI from Bergner et al. (1988), JHKLMN from Berrilli et al. (1992), and $Q$-band data from Simon (1974). In the mm/submm wavelength regime $(0.35-1.3 \mathrm{~mm})$ the dust continuum measurements are taken from Mannings (1994). At radio wavelengths $(6 \mathrm{~cm})$, MWC 297 has been observed by Drew et al. (1997). Existing NIR interferometric data for MWC 297 consist of two sets. IOTA $H$-band continuum data were presented by Millan-Gabet et al. (2001), and PTI $K$-band continuum data were published by Eisner et al. (2004, upper limits only). 


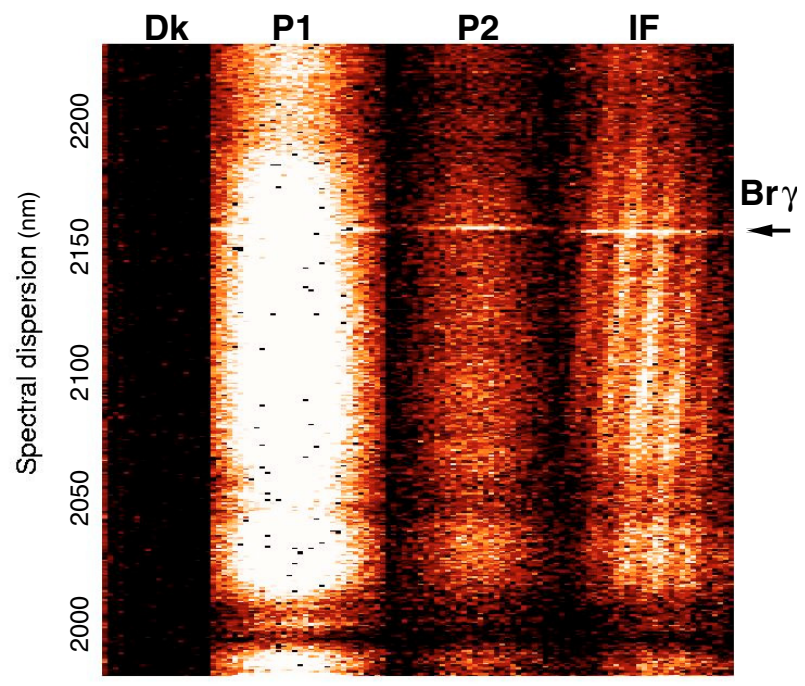

Fig. 1. Short-exposure image of the MWC 297 signal on the AMBER detector. $X$-axis corresponds to the spatial extension of the beams and $Y$-axis to the wavelength. First column (Dk) corresponds to the dark, the second (P1) and third (P2) ones are the beams from the first and second telescope resp., and finally the last column (IF) shows the fringes obtained by superposition of the two beams. The bright row is the $\mathrm{Br} \gamma$ line at $2165.6 \mathrm{~nm}$.

\section{AMBER data processing}

\subsection{Raw data}

AMBER follows the standard data flow system implemented at ESO/VLT. During data acquisition, the software records the images of the spectrally dispersed fringes as well as those of the telescope beams. Figure 1 shows a typical image retrieved from the detector subsystem ${ }^{1}$. From the left to the right, the first column contains an estimation of the dark, the second and third ones are the spectrophotometric measurements from each telescope in order to evaluate their respective contribution to the interferogram, and the last one is the spectrally dispersed fringe pattern, corresponding to the interference between the two telescope beams. Each exposure (or file) consists of one hundred of these elementary frames.

One can notice the bright line near the top of the figure and the dark lines at the bottom. They correspond to the $\mathrm{Br} \gamma$ emission line at $2.1656 \mu \mathrm{m}$ and to the telluric absorption lines around 2.05 and $2.08 \mu \mathrm{m}$ respectively.

\subsection{Data reduction}

Since we are still in an early stage of AMBER observations, we decided to use two different methods to extract the raw visibilites. We present and discuss them briefly.

- The standard data reduction method developed and optimized for AMBER is called P2VM for Pixel-To-Visibilities Matrix (Millour et al. 2004; Tatulli et al. 2007). The P2VM is a linear matrix method which derives raw visibilities from AMBER data for each spectral channel. The P2VM is computed after an internal calibration procedure which is performed every time the instrument configuration changes. The complex coherent fluxes ${ }^{2}$ are given by the product of the fluxes measured on each pixel of the detector by this P2VM.

1 As a matter of fact, this image is not exactly how it appears on the detector, since the four columns come from more widely separated regions of the detector.

${ }^{2}$ Coherent flux is the degree of complex coherence not yet normalized by the flux.
Table 2. Calibrated visibilities obtained with different algorithms and data selection.

\begin{tabular}{lllcc}
\hline \hline Algorithm & Selection & DIT $(\mathrm{ms})$ & $V_{\text {line }}$ & $V_{\text {cont }}$ \\
\hline P2VM & 35\% best exposures & 107 & $0.31 \pm 0.02$ & $0.58 \pm 0.03$ \\
P2VM & 25\% best exposures & 31 & $0.33 \pm 0.03$ & $0.57 \pm 0.05$ \\
P2VM & $10 \%$ best frames & 107 & $0.27 \pm 0.02$ & $0.41 \pm 0.04$ \\
P2VM & $10 \%$ best frames & 31 & $0.36 \pm 0.03$ & $0.57 \pm 0.06$ \\
FT & $10 \%$ best frames & 107 & $\leq 0.31$ & $0.39 \pm 0.05$ \\
FT & $10 \%$ best frames & 31 & $\leq 0.39$ & $0.45 \pm 0.05$ \\
\hline
\end{tabular}

We estimate the visibility for each spectral channel by integrating the square amplitude of the complex coherent fluxes over a certain number of frames and then by normalizing it by the total flux obtained for the same set of frames. The number of frames used can vary between 1 and the total number of frames in one exposure. This parameter hereafter is called the binning.

- A Fourier transform (FT) technique (see Ohnaka et al. 2003, Appendix A) has been used. The advantage is that it does not depend on the internal calibration of the instrument, described above. To enhance the signal, we applied a sliding average of seven spectral channels which has no effect on the continuum visibilities, but allows us to retrieve only upper limits of the visibility in the $\mathrm{Br} \gamma$ line. The visibility is computed using power spectral densities averaged over a variable number of frames.

Both methods compute squared visibilities and therefore an important step is to subtract the visibility bias, i.e. the part of the visibility due to photon and read-out noises. Because of the presence of vibrations, we are not completely sure that this bias is perfectly estimated and therefore we use very conservative errors.

\subsection{Data selection}

Because of the specific conditions of our observations, one critical step in the processing is the selection of the best data within the whole set. We based our selection of individual frames or exposures on the value of the visibility signal-to-noise ratio (SNR).

We used two different methods of selection based either on exposure or frame selection.

- The selection over the exposures consists of selecting a certain fraction of the best exposures. The visibilities for each exposure are computed using all frames. The final value is the average of the visibilities over the selected exposures weighted by the statistical errors.

- The selection over the frames consists of selecting a certain fraction of the best frames within each exposure acquired on the object. The final value of the visibility is the average of the visibilities of all selected frames weighted by their errors.

The exposure selection has been used only with the P2VM method whereas the frame selection has been used with both reduction methods. In all cases, the selection has been applied to the object and its calibrator and the resulting calibrated visibilities are summarized in Table 2.

The threshold for the selection process is an important parameter. Selecting with a strong criteria (e.g. $\leq 5 \%$ ) would not provide reliable statistics for the biases on the visibility squared. Selection with a soft criteria (e.g. above $\geq 50 \%$ ) may retain influences by a reduced fringe contrast due to telescope vibrations. Therefore we chose $10 \%$ for the frame selection, and 25\% (resp. $35 \%$ ) for the exposure selection of the $31 \mathrm{~ms}$ (resp. $107 \mathrm{~ms}$ ) 


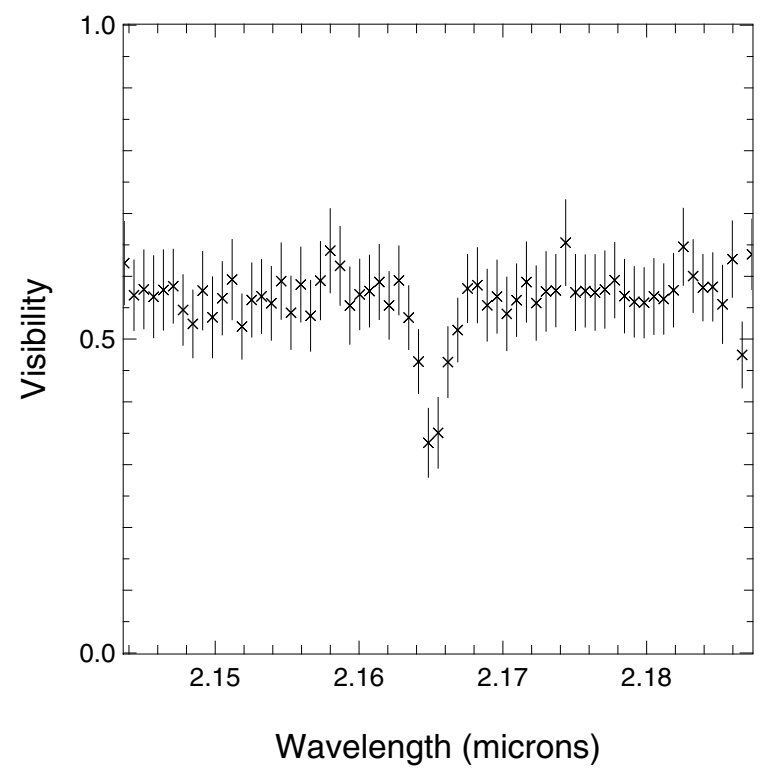

Fig. 2. Spectral dependence of the visibility as measured with AMBER for MWC 297 around the $\operatorname{Br} \gamma$ line.

data set because of the small number of exposures (see log of observations).

\section{Results}

\subsection{Continuum visibilities}

The continuum visibilities computed with the different algorithms and selection schemes are summarized in the right column of Table 2. We find discrepancies between the two methods that we are not able to explain at this very early stage of VLTI and AMBER operation. These discrepancies might be related to the presence of vibrations in the VLT UT coude trains as mentioned in Sect. 2, or to remaining imperfection in the estimation or subtraction of the biases. We propose to take the following continuum visibility with a relatively large error: $V_{\text {cont }}=$ $0.50_{-0.10}^{+0.08}$. This confirms that the environment of MWC 297 is spatially resolved at the level of a few milliarcseconds (Eisner et al. 2004). The uniform disk diameter corresponding to the visibility measured is $7.0 \pm 0.9$ mas, corresponding to $1.75 \pm 0.23 \mathrm{AU}$ at $250 \mathrm{pc}$.

\subsection{Spectral variation of the visibilities}

All methods and selection schemes give consistent visibilities for the $\mathrm{Br} \gamma$ emission line at $2.1656 \mu \mathrm{m}$ : $V_{\text {line }}=0.33 \pm 0.06$ (see Table 2). Figure 2 shows the variation of the visibility with wavelength for the $31 \mathrm{~ms}$ data. For this figure, the continuum and line visibilities were calculated using the P2VM and the best exposure selection criterion.

The visibility of the pure $\mathrm{Br} \gamma$ emission must be corrected for the influence of the continuum by the following relationship:

$V_{\mathrm{Br} \gamma}=\frac{F_{\text {line }} V_{\text {line }}-F_{\text {cont }} V_{\text {cont }}}{F_{\text {line }}-F_{\text {cont }}}$

where $F_{\mathrm{Br} \gamma}=F_{\text {line }}-F_{\text {cont }}$. Since the ratio of the line flux to the continuum flux is $F_{\text {line }} / F_{\text {cont }}=2.2$, the visibility of the region emitting the $\mathrm{Br} \gamma$ line is $V_{\mathrm{Br} \gamma}=0.19 \pm 0.03$, corresponding to a uniform disk diameter of $9.9 \pm 0.3$ mas or $2.5 \pm 0.08 \mathrm{AU}$ at $250 \mathrm{pc}$. Therefore, the size of the $\mathrm{Br} \gamma$ emitting region is $40 \%$ larger (in terms of uniform disk diameter) than the size of the region contributing to the continuum.

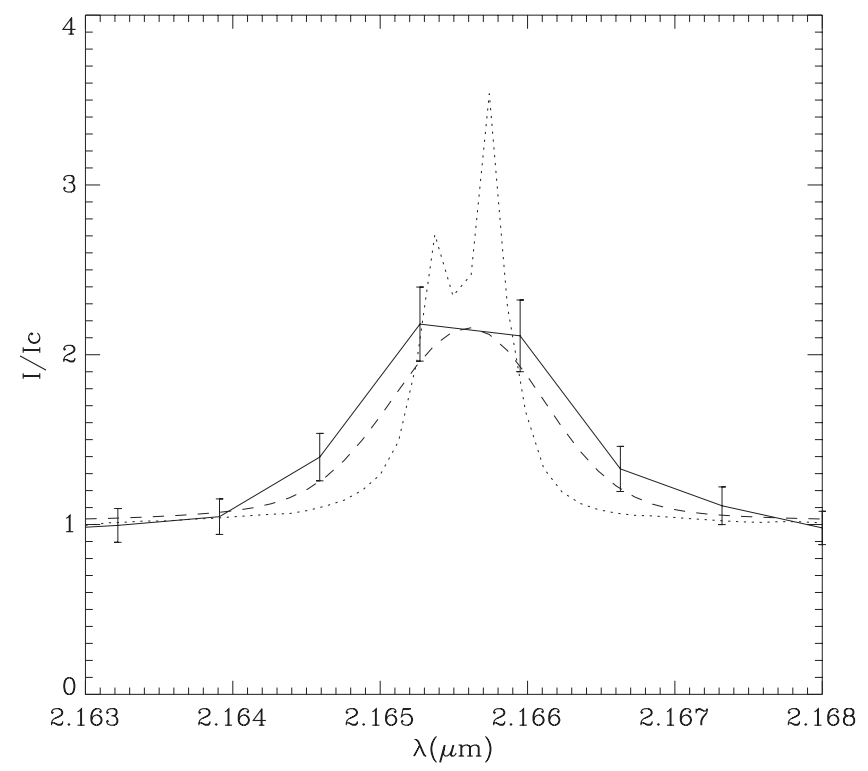

Fig. 3. Comparison of $\operatorname{Br} \gamma$ observed with AMBER (solid line) and ISAAC (dotted line). The dashed line corresponds to the ISAAC spectrum convolved by the response function of AMBER.

\subsection{ISAAC spectrum}

The AMBER spectrum at medium resolution $(\mathcal{R} \approx 1500)$ contains the $\mathrm{Br} \gamma$ line. Its profile has been compared to the ISAAC high spectral resolution $(\mathcal{R} \approx 8900)$ spectra obtained at nearly the same epoch in May and July 2004 respectively. A comparison between the two lines is given in Fig. 3. The equivalent width of the AMBER spectrum is $2.14 \mathrm{~nm}$ and is compatible with the $1.58 \mathrm{~nm}$ measured from the ISAAC spectrum given the spectral variability of MWC 297. We note that the resolved infrared $\mathrm{Br} \gamma$ emission lines presented in Murdoch \& Drew (1994) are symmetric rather than double-peaked.

The ISAAC spectral resolution is higher than the one achieved with AMBER. Nevertheless, in order to compare our two data sets the ISAAC line profile was convolved with a Gaussian function corresponding to the AMBER spectral resolution. The shape of both profiles are quite similar (see Fig. 3) but since the higher spectral resolution of the ISAAC spectrum provides more detail on the kinematics within the circumstellar envelope, we have used, in the following, the ISAAC Br $\gamma$ line profile in order to constrain the outflowing wind model (Sect. 5.2).

\section{Modeling}

In this section we present the modeling of the large body of interferometric, spectroscopic and photometric data that exists for MWC 297. The modeling is done by applying two different codes, one for an optically thick disk and one for a stellar wind. The disk code is designed to model the continuum radiation, whereas the stellar wind code reproduces the strong emission lines. Figure 4 represents a sketch of the combined model, where the optically thick disk and the outflowing wind are spatially independent.

\subsection{Continuum radiation: optically thick disk}

The disk model is based on the simple model already used by Malbet \& Bertout (1995) and Malbet et al. (2005). This disk model consists of an axisymmetric radial analytic disk structure which is heated both by stationary accretion and stellar 


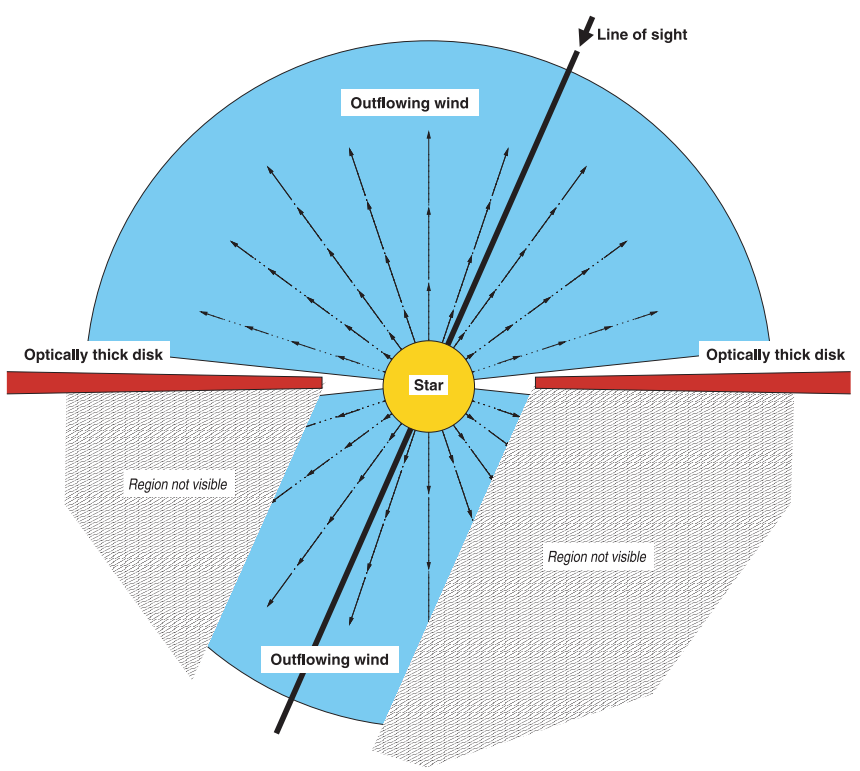

Fig. 4. Sketch of the model including an optically thick disk and an outflowing wind (edge-on view). The receding part of the wind is only partly visible because of the screen made by the optically thick disk.

irradiation. The disk is in hydrostatic equilibrium and non selfgravitating. The accretion flux follows the standard power law for a viscous disk (Lynden-Bell \& Pringle 1974; Shakura \& Sunyaev 1973). The emitted continuum flux is produced by the emission of optically thick but geometrically thin black-body radiating rings. It produces an SED, and its spatial distribution can be Fourier transformed, which reduces to a Hankel transform for the radial symmetry of a disk, to obtain interferometric visibilities.

The model effectively has five free parameters: the accretion rate $\dot{M}_{\text {acc }}$, the inclination $i$, the position angle $\theta$, and the distances of the inner and outer edge of the disk, $R_{\text {in }}$ and $R_{\text {out }}$ respectively. Modeling is strongly constrained by the accurately determined properties of the central star by Drew et al. (1997): a stellar radius of $6.12 R_{\odot}$, a mass of $10 M_{\odot}$, an effective temperature of $23,700 \mathrm{~K}$ for a distance of $250 \mathrm{pc}$ with a visual extinction of $A_{\mathrm{V}}=8 \mathrm{mag}$. The outer edge of the disk is strongly constrained by the $\mathrm{mm} / \mathrm{submm}$ data point: Mannings (1994) finds an outer disk radius of $\simeq 60 \mathrm{AU}$. In short, simultaneous fitting of the SED and the visibilities lead to balancing out the mass accretion rate, inner disk radius and the inclination. In practice, this is done iteratively by first fitting the SED, with $i$ and $\theta$ set to zero. We deem the $R$ - and $I$-band continuum measurements to be not reliable given the very strong $\mathrm{H} \alpha$ emission; these two are therefore not taken into account in the fit. In the first step we obtain $\dot{M}_{\text {acc }}$ and $R_{\text {in }}$. These are then used in a separate fit of the visibilities as function of $i$ and $\theta$. The results are used again for the first step and this procedure is repeated until convergence. In Table 3 we list the obtained best-fit model values. The modeled SED and visibilities are found to be in good agreement with the observed values, as shown in Fig. 5.

We probed the sensitivity of these fits by varying the central star parameters, according to the uncertainties given by Drew et al. (1997). They derived half a spectral subtype uncertainty, and a distance error of $50 \mathrm{pc}$. Fitting the SED using the extreme values for the central star, we find the uncertainties quoted in Table 3 . The mass accretion rate is especially far from being well determined. If the central star would be of type B2 at
Table 3. The best-fit accretion disk model parameters found by simultaneous fitting of SED and visibilities.

\begin{tabular}{ll}
\hline \hline Accretion rate $\left(\dot{M}_{\text {acc }}\right)$ & $0-1 \times 10^{-5} M_{\odot} \mathrm{yr}^{-1}$ \\
Inner radius $\left(R_{\text {in }}\right)$ & $0.5 \pm 0.1 \mathrm{AU}$ \\
Outer radius $\left(R_{\text {out }}\right)$ & $55 \pm 5 \mathrm{AU}$ \\
Inclination $(i)$ & $15 \pm 5^{\circ}$ \\
Position angle $(\theta)$ & $56 \pm 7^{\circ}$ \\
\hline
\end{tabular}

a distance of $200 \mathrm{pc}$, the required mass accretion rate is only $\sim 10^{-7} M_{\odot} \mathrm{yr}^{-1}$.

\subsection{Emission lines: optically thin outflowing wind}

In our model, the emission lines are produced in a circumstellar gas envelope. In order to model this line profile and the corresponding visibilities, we have used the SIMECA code (Stee \& de Araujo 1994; Stee et al. 1995). This code computes classical observables, i.e. spectroscopic and photometric ones but also intensity maps in Balmer lines and in the continuum in order to obtain theoretical visibility curves. The main assumptions are that (i) the envelope is axisymmetric with respect to the rotational axis; (ii) no meridian circulation is allowed, (iii) the physics of the polar regions are well represented by a CAK type stellar wind model (Castor et al. 1975). The solutions for all stellar latitudes are obtained by introducing a parametrized model (power of sinus function) constrained by the spectrally resolved interferometric data. Depending on the value of the chosen terminal velocity at the equator, the equatorial region can be dominated either by Keplerian rotation or by expansion.

Since the SIMECA code was originally developed to model the circumstellar environment of classical Be stars, we had to modify the code in order to interface SIMECA with the optically thick disk model described previously. We have implemented three changes (the equations describing the wind model are recalled in Appendix A):

1. The wind is no longer computed from the equator to the pole, but the computation occurs in a bipolar cone defined by a minimal angle allowing the disk to be present (see sketch in Fig. 4). The disk model tells us that the opening angle is between $1.8^{\circ}$ at the inner radius and up to $3.2^{\circ}$ at the outer radius. We used a minimum angle of $4^{\circ}$. Therefore the equatorial terminal velocity corresponds to the terminal velocity at this minimal angle from the equatorial plane at the interface between the accretion disk and the stellar wind.

2. The disk hides the receding part of the wind. In Fig. 4, the part of the wind which is not visible by the observer is not taken into account in the outgoing flux.

3. Although the disk emission contributes less than $1 \%$ compared to the star flux in the visible (i.e. also in the $\mathrm{H} \alpha$ and $\mathrm{H} \beta$ lines) and can be neglected, at $2.1656 \mu \mathrm{m}$ the disk emission is 6.4 times larger than the stellar flux. This contribution is taken into account in addition to the excess due to free-free emission from the outflowing gas when computing continuum normalized intensities and also for the computation of the visibilities.

To be consistent with the disk model (although it might not be fully physical), we also use the same temperature and rotational velocity laws, respectively $T(r) \propto r^{-3 / 4}$ and a Keplerian rotation.

After running hundreds of simulations in order to constrain the physical parameters of the wind and to test the geometrical and kinematic hypothesis described later in Sect. 6.2, we 

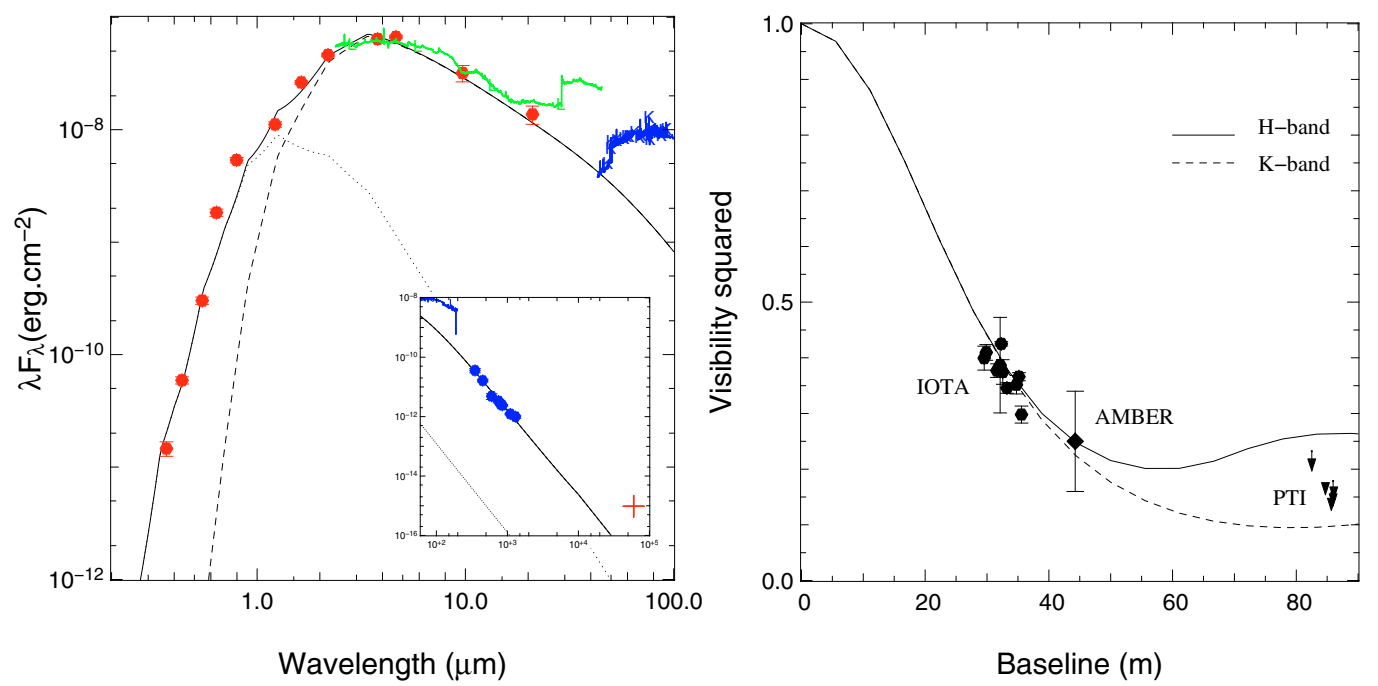

Fig. 5. Result from the optically thick disk model. Left panel: observed and modeled SED for MWC 297. The full dots are the continuum measurements taken from Pezzuto et al. (1997), also included are the ISO SWS/LWS spectra. Dotted line is the star, dashed line the accretion disk, and the full line the resulting total flux of the model. Right panel: resulting best-fit model radial squared visibilities compared with continuum squared visibilities observed with AMBER, IOTA and PTI. Full line and IOTA data are in the $H$-band, dashed line and AMBER/PTI are in the $K$-band. PTI values are upper limits.

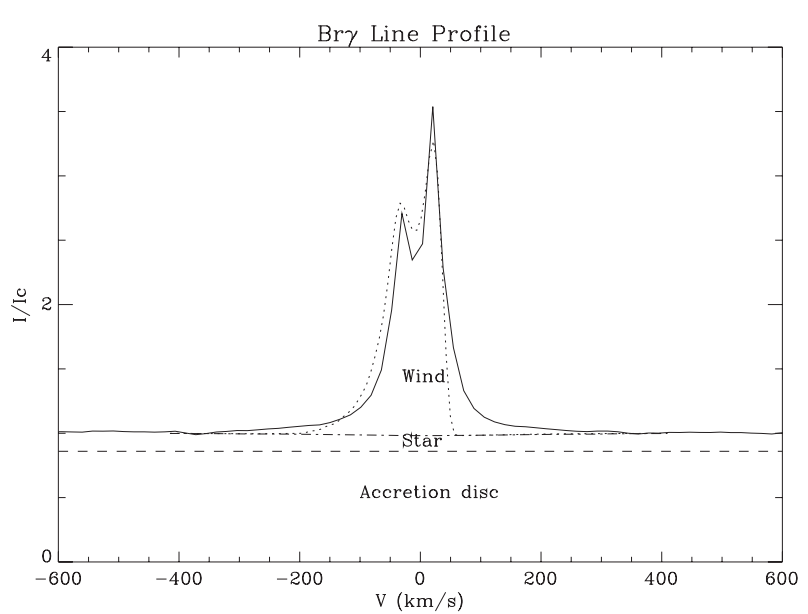

Fig. 6. Double peaked Br $\gamma$ profile observed by ISAAC (full line) and modeled with the outflowing wind model (dotted line). We have also plotted the cumulative contribution of the accretion disk (dashed line) and of the star (dashed-dotted).

find a successful simultaneous fit to the ISAAC Br $\gamma$ line profile (see Fig. 6) as well as $\mathrm{H} \alpha$ and $\mathrm{H} \beta$ profiles compatible with Drew's observations. The best-fit model parameters are given in Table 4. The outflowing wind model successfully reproduces the AMBER measured drop in visibility across the $\operatorname{Br} \gamma$ line as shown in Fig. 7.

The computed $\mathrm{H} \alpha$ and $\mathrm{H} \beta$ intensities are respectively 130 and 11.5 compared to 120 and 11 obtained by Drew et al. (1997). This corresponds to an intensity ratio $I_{\mathrm{H} \alpha} / I_{\mathrm{H} \beta}$ of 11.3 compatible with the Drew ratio of 11 regarding the stellar variability and the non-simultaneity of the measurements. Moreover we were able to reproduce quite well the shape of these lines (i.e. width) and the double peaked emission of the $\operatorname{Br} \gamma$ line. The asymmetry of the two peaks of the $\mathrm{Br} \gamma$ line is also successfully reproduced by the introduction in the SIMECA code of the opacity of the disk (point 2 of SIMECA modifications). Nevertheless the agreement is not perfect in the red wing of the profile probably due to our ad-hoc way of linking of the wind and the disk.

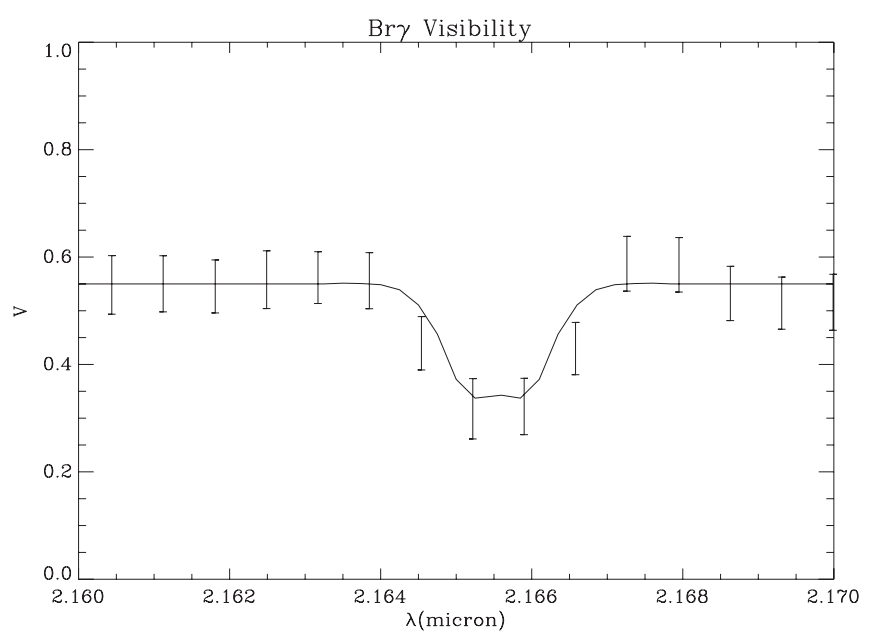

Fig. 7. The visibility observed with AMBER (points with error bars) and the one obtained from the outflowing wind model (full line).

Table 4. The best-fit model parameters for the outflowing wind model. Most parameters are self-explanatory (see Appendix A for details). $C_{1}$ is the ratio between the polar and equatorial mass flux; $m_{1}$ is the exponent of the mass flux law as a function of latitude; $m_{2}$ is the exponent of the latitude dependent terminal velocity law.

\begin{tabular}{ll}
\hline \hline Photospheric density & $1 \pm 0.5 \times 10^{12} \mathrm{~cm}^{-3}$ \\
Equatorial rotational velocity & $400 \pm 50 \mathrm{~km} \mathrm{~s}^{-1}$ \\
Polar terminal velocity & $600 \pm 50 \mathrm{~km} \mathrm{~s}^{-1}$ \\
Terminal velocity above disk & $70 \pm 20 \mathrm{~km} \mathrm{~s}^{-1}$ \\
Polar mass flux & $3.2 \pm 0.2 \times 10^{-9} M_{\odot} \mathrm{yr}^{-1}$ \\
$C_{1}$ & $0.25 \pm 0.05$ \\
$m_{1}$ & $30 \pm 10$ \\
$m_{2}$ & $10 \pm 2$ \\
Inclination angle $(i)$ & $25 \pm 5^{\circ}$ \\
\hline
\end{tabular}

The global shape of these lines and their intensities are very sensitive to the inclination angle. We were able to determine the inclination of $25 \pm 5^{\circ}$ from the fitting of the line profiles with 
the SIMECA code. We note that the wind parameters given in Table 4 are different to classical Be stars. The mass flux ratio $\left(C_{1}\right)$ is 0.25 whereas for Be stars the allowed range is between 10 and 100. Knowing that the density in the envelope is proportional to the mass flux and inversely proportional to the radial velocity we obtain that the gas density in the polar region is twice as small as the one at the interface between the accretion disk and the stellar wind. The highest mass loss occurs along the polar direction.

\section{Discussion}

We have presented extensive existing data and new AMBER and ISAAC data on the Herbig Be star MWC 297. We have simultaneously modeled interferometric and non-interferometric data. The continuum emission is generated by an optically thick disk heated by stellar irradiation and accretion, whereas optical and NIR emission lines originate in an outflowing stellar wind.

The modeling presented in the previous section, although rather successful, raises new questions on the physics of the circumstellar environment of intermediate-mass young stars. In this section, we discuss them by first addressing separate issues about the disk and the wind, and then those raised by the combined model.

\subsection{Physical conditions in the disk}

Our continuum observations placed in the context of young stellar objects allow us to bring new elements into the discussion of protoplanetary disk models.

\subsubsection{Reprocessing versus accretion in MWC 297}

The mass accretion rate is poorly constrained by the viscous accretion disk model. Firstly, this is because the viscosity in the accretion disk is quite poorly understood, and therefore the quoted accretion rate cannot be assessed with real certainty. Secondly, we obtain two orders of magnitude variation in the value of the accretion rate by varying the stellar parameters according to their uncertainties (see Sect. 5.1).

The ratio between the reprocessed energy from the stellar irradiation and the viscous heating due to the accretion along the disk varies from $4 \times$ down to $3 \times\left(\dot{M}_{\text {acc }} / 10^{-5} M_{\odot} \mathrm{yr}^{-1}\right)^{-1}$ from the inner part to the outer part of the disk. Therefore for accretion rates lower than $10^{-9} M_{\odot} \mathrm{yr}^{-1}$, the reprocessing energy is always dominant compared to the accretion energy. In fact the transition between the two types of flux in the disk lies near $\dot{M}_{\text {acc }} \simeq 3 \times 10^{-5} M_{\odot} \mathrm{yr}^{-1}$ for the most probable values of the stellar parameters.

This means that the disk is predominantly a reprocessing disk. However as pointed out by Lachaume et al. (2003, see their Fig. 4), the emerging flux might be dominated by the stellar heating and the vertical structure by the viscous heating at least for the inner radii (less than a few AUs).

\subsubsection{Inner radius of the disk}

The best fit of our disk model is found with an inner disk boundary at $0.5 \mathrm{AU}$. This result can be compared to the analysis of Millan-Gabet et al. (2001). With an assumption of $d=450 \mathrm{pc}$, these authors found a ring diameter of about $10 \mathrm{AU}$ and an inner disk radius of $0.9 \mathrm{AU}$. Our result is therefore consistent with their disk model since we have adopted the distance $d=250 \mathrm{pc}$ of Drew et al. (1997).

As already pointed out by other authors (Eisner et al. 2004; Monnier et al. 2005), the inferred inner disk radius for MWC 297 is not compatible with the dust evaporation distance from the star. Using the disk model with the "puffed up" inner rim (Dullemond et al. 2001; Isella \& Natta 2005), the inner radius of the disk would be located at about 3 AU from the star, even in the most favourable hypothesis that the dust evaporation temperature is $2000 \mathrm{~K}$. Moreover, MWC 297 seems not to be a special case, since this behavior is common to the other two early HBe stars (Z CMa A with a spectral type B0 and V 1685 Cyg with spectral type B3) observed with near infrared interferometers (Monnier et al. 2005). One possible explanation may be that the gas is the dominant source of absorption in the inner part of the disk, preventing dust grains from evaporating near to the star.

Using Rosseland gray opacities (Ferguson et al. 2005), we checked that the disk is always optically thick both for an accreting disk (accretion rate higher than $10^{-9} M_{\odot} \mathrm{yr}^{-1}$ ) or for a reprocessed disk (disk density at the disk edge higher than $1 \%$ of the typical value of the solar nebula3; Hayashi 1981; Davis 2005).

Even with the last assumption, the structure of the inner region of early $\mathrm{HBe}$ stars is not totally understood. The physical reason why the optically thick gas disk should be truncated at $0.5 \mathrm{AU}$ from the star is not clear.

\subsubsection{Ionization of the disk}

We estimate whether the circumstellar disk is susceptible to magnetohydrodynamical (MHD) instabilities, and thus if the disk is in the active accretion state by means of magnetorotational instabilities (MRI; Balbus \& Hawley 1991). Given the large inner radius of $0.5 \mathrm{AU}$, it is not expected that the disk is accreting, otherwise the region interior to the disk inner radius would quickly be filled. Indeed, in the previously discussed hydrodynamic case, we saw that the disk seems to be dominated by reprocessing.

Whether MHD instabilities in a given circumstellar disk operate or are suppressed can be estimated with the magnetic Reynolds number (see e.g. Gammie 1996). By setting the Reynolds number equal to one, a threshold disk ionization fraction, $n_{\mathrm{e}} / n_{\mathrm{H}}$, of $3 \times 10^{-13}$ is found. In this computation we adopted the same parameters for the MWC 297 star-disk system as in the previous sections. The ionization fraction threshold is essentially determined by the ionization rate and the particle density in the disk. Considering a high $\left(10^{-5} M_{\odot} \mathrm{yr}^{-1}\right)$ and a low $\left(10^{-9} M_{\odot} \mathrm{yr}^{-1}\right)$ mass accretion rate for MWC 297, we can use Fig. 1 of Inutsuka \& Sano (2005) to deduce that the required ionization rate in a dusty disk should range between $10^{-14}$ and $10^{-15} \mathrm{~s}^{-1}$ or between $10^{-10}$ and $10^{-11} \mathrm{~s}^{-1}$ respectively. Given that the cosmic ray ionization rate is close to $10^{-17} \mathrm{~s}^{-1}$, we conclude that cosmic rays alone are insufficient to incite and/or sustain the required fractional ionization for MRI to operate.

Since ultraviolet radiation is generally considered to be inefficient due to the very small attenuation length, X-ray ionization may play an important role in the ionization structure of the disk. The very strong X-ray activity reported for MWC 297 with ASCA (Hamaguchi et al. 2000) should be interpreted with care given the reasonably strong case for source confusion (Vink et al. 2005). An inner disk radius of $0.5 \mathrm{AU}$ is thus probably not susceptible to MHD instabilities. We conclude thus that from a

\footnotetext{
${ }^{3}$ Solar nebula density law used: $\Sigma_{S N}=1.7 \times 10^{3}(r / 1 \mathrm{AU})^{-1.5} \mathrm{~g} / \mathrm{cm}^{2}$.
} 

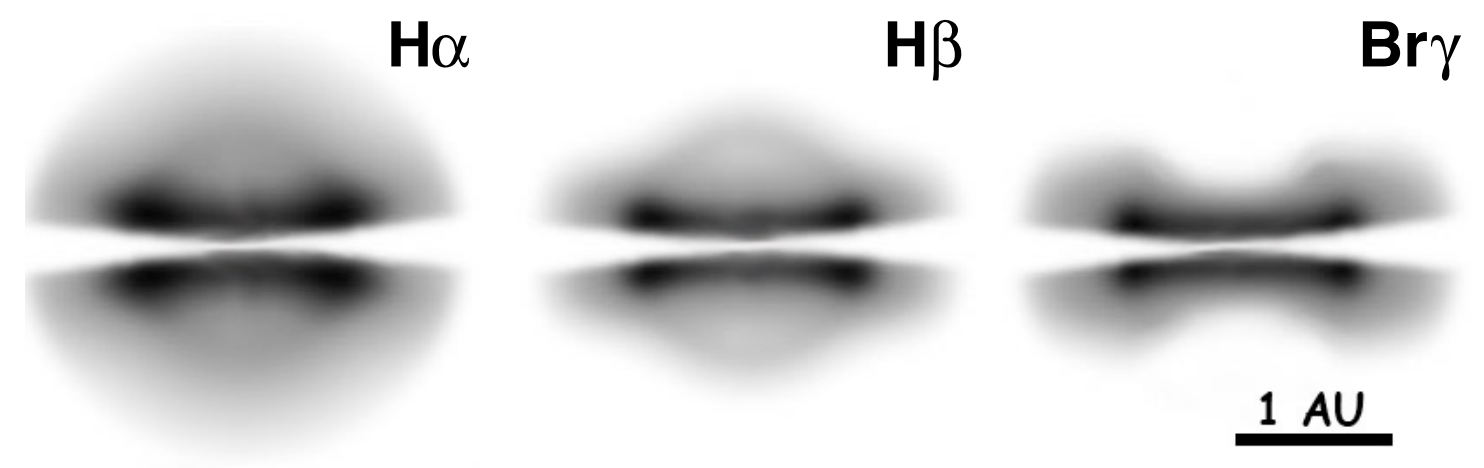

Fig. 8. Edge-on intensity maps of the wind in the computed $\mathrm{H} \alpha, \mathrm{H} \beta, \mathrm{Br} \gamma$ lines.

MHD and a hydrodynamic point of view an inert reprocessing disk may exist. We note however that if in the past the disk of MWC 297 was in fact a magnetically active disk in which MRI operated, that then the concomitant MRI turbulence could have sustained the ionization degree above the threshold level even in the presence of dust (Inutsuka \& Sano 2005).

\subsection{Geometry of the wind}

First, we investigate why the standard Be model of a rotating wind is unsuccessful in reproducing the MWC 297 data. In this model (Stee \& de Araujo 1994; Stee et al. 1995), the Br $\gamma$ emission would originate from a rotating flattened envelope with no or little radial expansion. We can test the consistency of this model by comparing the size of the emitting region given by the kinematics revealed by the splitting of the $\operatorname{Br} \gamma$ emission line into two peaks and the radius of this same region inferred by the drop in visibility in this $\mathrm{Br} \gamma$ line.

On the one hand, the projected rotational velocity of the orbiting material is given by the separation of the two $\mathrm{Br} \gamma$ peaks and is proportional to the maximum extension of the emitting region (Huang 1972; Hirata \& Kogure 1984). Using a rotational velocity law of the form $v(r)=v_{0}\left(r / R_{\star}\right)^{-x}$, where $v_{0}$ is the star rotational velocity at the equator, $r$ is the distance to the star, and $x$ is the exponent of the rotational velocity law, the separation between the two peaks is proportional to $2 v_{0} \sin i\left(r / R_{\star}\right)^{-x}$. Therefore the maximal radius of emission of the $\operatorname{Br} \gamma$ line is $\sim\left(2 v_{0} \sin i / d\right)^{1 / x}$, and the stellar parameters given by Drew et al. (1997) and a Keplerian rotation yield $\sim 140 R_{\star}$.

Since the typical radius of emission of the $\mathrm{Br} \gamma$ emission is $\sim 43 R_{\star}$ (see Sect. 4.2), the size of $140 R_{\star}$ derived from the Keplerian rotation of a rotating stellar Be wind is not consistent with our interferometric measurement.

In order to obtain an emitting zone radius compatible with our interferometric measurement we need to assume a rotational velocity of less than $250 \mathrm{~km} \mathrm{~s}^{-1}$ or a rotation law far from the Keplerian one. In total, we ran 50 models in our attempt to fit the $\mathrm{Br} \gamma, \mathrm{H} \alpha, \mathrm{H} \beta$ lines and the visibility in $\mathrm{Br} \gamma$ simultaneously using this type of wind model. None of these models was able to fit correctly the Br $\gamma$ line profile displayed in Fig. 3 nor the intensity ratio of the three hydrogen lines. In addition, the $\mathrm{H} \alpha$ and $\mathrm{H} \beta$ emission lines must form at least partially in a strong stellar wind to reproduce the $600 \mathrm{~km} \mathrm{~s}^{-1}$ line width in contrast to the $\mathrm{Br} \gamma$ line displaying a $60 \mathrm{~km} \mathrm{~s}^{-1}$ double-peaked profile. This is unlikely in the same environment.

The outflowing stellar wind coupled with an optically thick disk gives a better explanation of the kinematical and

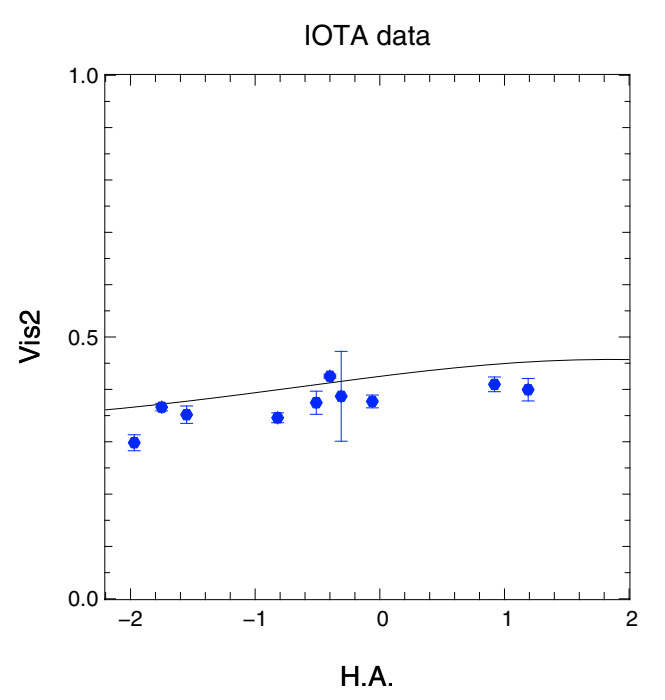

Fig. 9. Continuum $H$-band squared visibilities obtained with the disk model compared to the IOTA visibilities observed during the transit of the object over the sky with the IOTA S15N35 baseline.

geometrical morphology of MWC 297. In Fig. 8, we present the intensity maps of MWC 297 computed with our model seen edge-on in the three hydrogen lines in order to better localize the region of emission of the lines. The emission from $\mathrm{H} \alpha$ and $\mathrm{H} \beta$ originates from a large and somewhat spherical region where the velocity can reach up to $600 \mathrm{~km} \mathrm{~s}^{-1}$. The $\mathrm{Br} \gamma$ line is confined into a narrower region just above the optically thick disk where the velocity is dominated by the disk Keplerian rotation and a terminal velocity of $70 \mathrm{~km} \mathrm{~s}^{-1}$ (see parameters in Table 4). Murdoch \& Drew (1994) have already pointed out that the $\operatorname{Br} \alpha / \operatorname{Br} \gamma$ line flux ratio shows an increase at low velocity that cannot be interpreted as an outwardly-accelerated wind model.

\subsection{Disk and wind interaction in MWC 297}

Even if the disk and the wind have been modeled separately, these two physical phenomena need to be compared at least at the parameter level. We find a good correspondence between the inclinations deduced from the disk model $\left(i=15 \pm 5^{\circ}\right.$ from the visibility fit) and from the wind model $\left(i=25 \pm 5^{\circ}\right.$ from the asymmetry of the $\operatorname{Br} \gamma$ line peaks). The disk inclination is strongly constrained by the IOTA visibilities spread out over a large range of hour angle as shown in Fig. 9. The wind mass loss rate is smaller than the maximum disk accretion rate by several orders of magnitude, and therefore compatible with most 

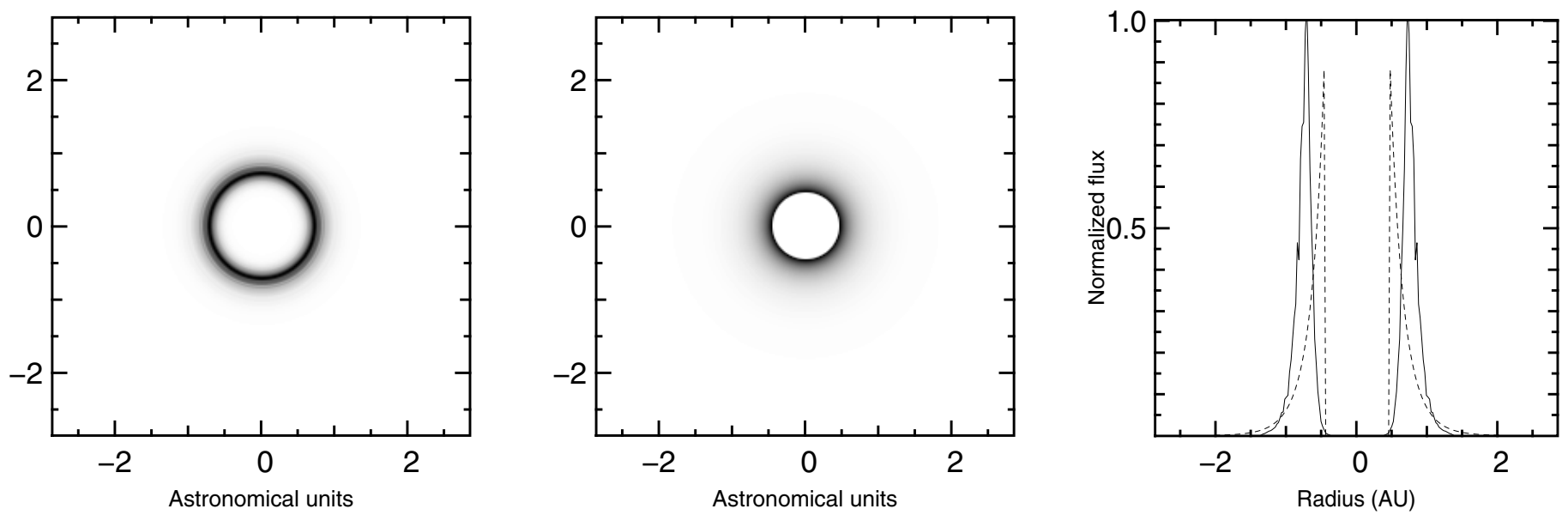

Fig. 10. Pole-on intensity maps of the wind $\mathrm{Br} \gamma$ emission (left panel) and of the $K$-band disk continuum emission (center panel). Right panel shows a radial cut of these intensity maps with the $\operatorname{Br} \gamma$ wind in solid line and the continuum disk in dashed line.

disk/wind theories but also with most observations of outflows and accretion activities. As shown in Sect. 6.1.3, the inner radius is barely ionized and thus will not contribute to the hydrogen lines, confirming our choice to model Br $\gamma$ only in the wind.

Figure 10 shows the pole-on intensity maps of the disk model in the continuum and of the wind in the Br $\gamma$ line, as well as their respective intensity profile. This is a graphical explanation of the visibilities observed by AMBER: the wind angular extension in the $\mathrm{Br} \gamma$ line is larger than the disk apparent size, and therefore the visibility is smaller within the line.

Can the result obtained with these observations constrain the nature of the wind? We recall that in protoplanetary disks, two main classes of disk wind models have been proposed depending on the geometry of the magnetic field lines.

- The disk wind initially proposed by Blandford \& Payne (1982) and studied in detail for example in Casse \& Ferreira (2000). In this theory the magnetic field is attached to the disk and the particles that accrete onto the star may be launched into a wind eventually collimated at a certain distance from the star.

- The X-wind of Shu et al. (1994) has a different geometry and originates at the corotation radius of the disk where all magnetic field lines are localized in the equatorial plane.

With the present data, we cannot even distinguish between a stellar wind and such disk winds since we are unable to recover a precise kinematical map of the wind. We note however that our model with Keplerian rotation does not respect the conservation of angular momentum and can only be sustained if additional MHD energy is injected in the wind. Increased spectral resolution with AMBER will help to answer the question, especially using the 10000 spectral resolution mode.

Thus, we can claim that the models of disk and wind are compatible and the combination of them is probably close to reality. A complete and self-consistent modeling of the environment is out of the scope of this paper but would allow us to better constrain the physical processes in action both in the disk and the wind, such as accretion and ejection, at least from the observational point of view.

\subsection{Inclination discrepancy}

The modeling presented in this paper does a reasonably good job in reproducing nearly all the observational data and produces fiducial physical parameters for the circumstellar environment of MWC 297. However, we derive an inclination of $\sim 20^{\circ}$ for the system, which is not consistent with a near edge-on orientation as proposed by Drew et al. (1997). The latter is inferred from the photospheric lines that indicate a $350 \mathrm{~km} \mathrm{~s}^{-1}$ projected rotational velocity. An inclination of $20^{\circ}$ would lead to a rotation above the break-up velocity.

The controversy becomes even more pronounced given the $\mathrm{H} \alpha$ spectro-polarimetric results by Oudmaijer \& Drew (1999). These authors find no effect across the line, a result consistent with a symmetric emission zone for $\mathrm{H} \alpha$. On the contrary, the morphology of the radio emission (Drew et al. 1997) favors a bipolar interpretation and again would point to a high inclination of the system, although not completely inconsistent with a symmetric geometry on smaller scales (Oudmaijer \& Drew 1999).

A possible reconciliation of the seemingly contradictory observables and derived physical parameters is a tidally induced warped geometry for the circumstellar disk (Terquem \& Bertout 1993) due to a stellar companion located at a distance of a few hundred AU. Recently, Vink et al. (2005) reported the presence of a close-by late-type companion at a projected distance of 850 AU. By converting the $H$-band magnitude of the Vink et al. (2005) companion star to a spectral type A2 V and by calculating the steady-state deformation of the MWC 297 circumstellar disk due to the star at $850 \mathrm{AU}$, (Eq. (25) of Terquem \& Bertout 1993), we find a deformation of less than $1 / 1000$ even at the edge of the disk at $60 \mathrm{AU}$. Clearly this star would not be the right candidate, but we feel that the possibility of a tidally deformed disk may not yet be ruled out given the many companion stars found near MWC 297 (Testi et al. 1999; Vink et al. 2005). An even closer companion cannot be excluded, a hint of which may be the CHANDRA X-rays found coincident with the position of MWC 297.

\section{Summary and perspectives}

We have presented the first spatially and spectrally resolved observations of the disk/wind interaction in the young stellar system MWC 297 with the VLT interferometer equipped with the instrument AMBER. The unique spectral capability of AMBER has allowed us to measure for the first time the visibility in the $\operatorname{Br} \gamma$ emission line in a young stellar object and to compare it to the continuum visibility. The continuum visibility in the $K$-band drops from 0.50 to 0.33 in the $\mathrm{Br} \gamma$ emission line of MWC 297. 
The spectrum obtained with AMBER is consistent with a double peaked spectrum observed with ISAAC on the VLT, where the peaks are separated by approximately $60 \mathrm{~km} \mathrm{~s}^{-1}$.

We have successfully modeled the circumstellar environment of MWC 297 using an optically thick geometrically thin disk and an outflowing stellar radial wind having an increasing outflowing velocity starting from the surface of the disk up to the pole. This combined model is able to reproduce many observational features like the continuum visibilities measured by AMBER as well as the visibilities in the $\mathrm{Br} \gamma$ line, together with the shape of the SED over more than three orders of magnitude of the wavelengths, the broad-band visibilities obtained by other infrared interferometers as well as the $\mathrm{H} \alpha, \mathrm{H} \beta$ and $\mathrm{Br} \gamma$ line profiles.

We have discussed our result in the light of more sophisticated models. We showed that the inner radius is not determined by the dust sublimation distance, and is unlikely ionized by cosmic rays only. The disk flux is mainly driven by stellar reprocessing although we cannot rule out that the accretion process may play a role in the vertical structure. We have shown that our AMBER observations interpreted by our model predict emission of the $\mathrm{H} \alpha$ and $\mathrm{H} \beta$ lines in the polar regions whereas the $\mathrm{Br} \gamma$ emission arises mainly from the region just above the surface of the disk. AMBER continuum and line observations both point toward a system inclination of approximatively $20^{\circ}$.

We are not yet able to constrain the exact nature of the wind and the type of connection to the disk, but we expect that future observations with AMBER will bring new elements in our understanding of MWC 297. The vibrations in the VLTI UT optical train should be diagnosed and fixed in the short term and the VLTI equipped with a fringe tracker, allowing AMBER to be operated at its highest spectral resolution $(\sim 10000)$ which will give new kinematical information on this interesting and intriguing region around MWC 297. Another direction of investigation would be to carry out AMBER observations with three telescopes in order to measure the closure phase with different baseline configurations, and therefore measure possible departure from centro symmetry of the material around MWC 297.

Acknowledgements. We are grateful to R. Millan-Gabet and J. Eisner who kindly provided their IOTA and PTI measurements. This work is based on observations made with the European Southern Observatory telescopes. The commissioning data can be retrieved from the ESO Science Archive Facility within the programme 60.A-9054(A).

The AMBER project ${ }^{4}$ was founded by the French Centre National de la Recherche Scientifique (CNRS), the Max Planck Institute für Radioastronomie (MPIfR) in Bonn, the Osservatorio Astrofisico di Arcetri (OAA) in Firenze, the French Region "Provence Alpes Côte D'Azur" and the European Southern Observatory (ESO). The CNRS funding has been made through the Institut National des Sciences de l'Univers (INSU) and its Programmes Nationaux (ASHRA, PNPS, PNP).

The OAA co-authors acknowledge partial support from MIUR grants to the Arcetri Observatory: A LBT interferometric arm, and analysis of VLTI interferometric data and From Stars to Planets: accretion, disk evolution and planet formation and from INAF grants to the Arcetri Observatory Stellar and Extragalactic Astrophysics with Optical Interferometry. C. Gil work was supported in part by the Fundação para a Ciência e a Tecnologia through project POCTI/CTE-AST/55691/2004 from POCTI, with funds from the European program FEDER.

The preparation and interpretation of AMBER observations benefit from the tools developed by the Jean-Marie Mariotti Center for optical interferometry $\mathrm{JMMC}^{5}$ and from the databases of the Centre de Données Stellaires (CDS) and of the Smithsonian/NASA Astrophysics Data System (ADS).

${ }^{4}$ The structure and members of the AMBER Consortium can be found in the website: http://amber.obs.ujf-grenoble.fr

5 The JMMC is a center providing software tools for optical interferometry described at the website: http://www.jmmc.fr
The data reduction software amdlib is freely available on the AMBER site http://amber.obs.ujf-grenoble.fr. It has been linked to the public domain software Yorick $^{6}$ to provide the user-friendly interface ammyorick.

\section{Appendix A: Wind model equations}

Following Stee \& de Araujo (1994) and Stee et al. (1995), the mass flux is parametrized as:

$\Phi(\theta)=\Phi_{\text {pole }}+\left[\left(\Phi_{\text {eq }}-\Phi_{\text {pole }}\right) \sin ^{m_{1}}(\theta)\right]$,

where $m_{1}$ is the first free parameter which describes the variation of the mass flux from the pole to the equator. The ratio between the equatorial and polar mass flux is $C_{1}=\Phi_{\text {eq }} / \Phi_{* \text { pole }}$. The values of $C_{1}$ are typically between $10^{1}$ and $10^{4}$ (Lamers \& Waters 1987). Equation (A.1) can be rewritten as:

$\Phi(\theta)=\Phi_{\text {pole }}\left[1+\left(C_{1}-1\right) \sin ^{m_{1}}(\theta)\right]$.

The expansion velocity field is given by:

$v_{r}(r, \theta)=V_{o}(\theta)+\left[V_{\infty}(\theta)-V_{o}(\theta)\right]\left(1-\frac{R}{r}\right)^{\gamma}$,

with $\gamma=0.86$ and

$V_{o}(\theta)=\frac{\Phi(\theta)}{\rho_{0}}=\frac{\Phi_{\text {pole }}\left[1+\left(C_{1}-1\right) \sin ^{m_{1}}(\theta)\right]}{\rho_{0}}$.

The second free parameter $m_{2}$ is introduced in the expression of the terminal velocity as a function of the stellar latitude:

$V_{\infty}(\theta)=V_{\infty}($ pole $)+\left[V_{\infty}(\right.$ eq $)-V_{\infty}($ pole $\left.)\right] \sin ^{m_{2}}(\theta)$.

Finally the density distribution in the envelope is given by the equation of mass conservation:

$\rho(r, \theta)=\frac{\Phi(\theta)}{\left(\frac{r}{R}\right)^{2} v_{r}(r, \theta)}$.

\section{References}

Balbus, S. A., \& Hawley, J. F. 1991, ApJ, 376, 214

Bergner, Y. K., Kozlov, V. P., Krivtsov, A. A., et al. 1988, Astrophysics, 28, 313 Berrilli, F., Corciulo, G., Ingrosso, G., et al. 1992, ApJ, 398, 254

Blandford, R. D., \& Payne, D. G. 1982, MNRAS, 199, 883

Casse, F., \& Ferreira, J. 2000, A\&A, 353, 1115

Castor, J. I., Abbott, D. C., \& Klein, R. I. 1975, ApJ, 195, 157

Davis, S. S. 2005, ApJ, 627, L153

di Francesco, J., Evans, N. J., Harvey, P. M., Mundy, L. G., \& Butner, H. M. 1994, ApJ, 432, 710

Drew, J. E., Busfield, G., Hoare, M. G., et al. 1997, MNRAS, 286, 538

Dullemond, C. P., Dominik, C., \& Natta, A. 2001, ApJ, 560, 957

Eisner, J. A., Lane, B. F., Hillenbrand, L. A., Akeson, R. L., \& Sargent, A. I. 2004, ApJ, 613, 1049

Ferguson, J. W., Alexander, D. R., Allard, F., et al. 2005, ApJ, 623, 585

Gammie, C. F. 1996, ApJ, 457, 355

García Lopez, R., Natta, A., Testi, L., \& Habart, E. 2006, A\&A, 459, 837 Hamaguchi, K., Terada, H., Bamba, A., \& Koyama, K. 2000, ApJ, 532, 1111

Hayashi, C. 1981, Prog. Theor. Phys. Suppl., 70, 35

Herbig, G. H. 1960, ApJS, 4, 337

Hillenbrand, L. A., Strom, S. E., Vrba, F. J., \& Keene, J. 1992, ApJ, 397, 613

Hirata, R., \& Kogure, T. 1984, Bull. Astron. Soc. India, 12, 109

Huang, S. 1972, ApJ, 171, 549

Inutsuka, S., \& Sano, T. 2005, ApJ, 628, L155

Isella, A., \& Natta, A. 2005, A\&A, 438, 899

Kenyon, S. J., Calvet, N., \& Hartmann, L. 1993, ApJ, 414, 676

6 http://yorick. sourceforge.net 
Lachaume, R., Malbet, F., \& Monin, J.-L. 2003, A\&A, 400, 185

Lamers, H. J. G. L. M., \& Waters, L. B. F. M. 1987, A\&A, 182, 80

Lynden-Bell, D., \& Pringle, J. E. 1974, MNRAS, 168, 603

Malbet, F., \& Bertout, C. 1995, A\&AS, 113, 369

Malbet, F., Lachaume, R., Berger, J.-P., et al. 2005, A\&A, 437, 627

Mannings, V. 1994, MNRAS, 271, 587

Millan-Gabet, R., Schloerb, F. P., \& Traub, W. A. 2001, ApJ, 546, 358

Millour, F., Tatulli, E., Chelli, A., et al. 2004, in New Frontiers in Stellar Interferometry, ed. W. A. Traub, Proc. SPIE, 5491, 1222

Miroshnichenko, A., Ivezić, Ž., Vinković, D., \& Elitzur, M. 1999, ApJ, 520, L115

Monnier, J. D., \& Millan-Gabet, R. 2002, ApJ, 579, 694

Monnier, J. D., Millan-Gabet, R., Billmeier, R., et al. 2005, ApJ, 624, 832

Murdoch, K. A., \& Drew, J. E. 1994, in ASP Conf. Ser. 62, The Nature and Evolutionary Status of Herbig Ae/Be Stars, 377

Natta, A., Prusti, T., Neri, R., et al. 2001, A\&A, 371, 186

Ohnaka, K., Beckmann, U., Berger, J.-P., et al. 2003, A\&A, 408, 553

Oudmaijer, R. D., \& Drew, J. E. 1999, MNRAS, 305, 166

Petrov, R. G., Malbet, F., Weigelt, G., et al. 2007, A\&A, 464, 1

Pezzuto, S., Strafella, F., \& Lorenzetti, D. 1997, ApJ, 485, 290

Shakura, N. I., \& Sunyaev, R. A. 1973, A\&A, 24, 337

Shu, F., Najita, J., Ostriker, E., et al. 1994, ApJ, 429, 781

Simon, T. 1974, AJ, 79, 1054

Stee, P., \& de Araujo, F. X. 1994, A\&A, 292, 221

Stee, P., de Araujo, F. X., Vakili, F., et al. 1995, A\&A, 300, 219

Tatulli, E., Millour, F., Chelli, A., et al. 2007, A\&A, 464, 29

Terquem, C., \& Bertout, C. 1993, A\&A, 274, 291

Testi, L. Palla, F, \& Natta, A. 1999, A\&A, 342, 515

Tuthill, P. G., Monnier, J. D., \& Danchi, W. C. 2001, Nature, 409, 1012

Vink, J. S., Drew, J. E., Harries, T. J., \& Oudmaijer, R. D. 2002, MNRAS, 337, 356

Vink, J. S., O’Neill, P. M., Els, S. G., \& Drew, J. E. 2005, A\&A, 438, L21

1 Laboratoire d'Astrophysique de Grenoble, UMR 5571 Université Joseph Fourier/CNRS, BP 53, 38041 Grenoble Cedex 9, France e-mail: Fabien. Malbet@obs.ujf-grenoble.fr
2 Max-Planck-Institut für Radioastronomie, Auf dem Hügel 69, 53121 Bonn, Germany

3 Laboratoire Gemini, UMR 6203 Observatoire de la Côte d'Azur/CNRS, BP 4229, 06304 Nice Cedex 4, France

${ }^{4}$ Laboratoire Universitaire d'Astrophysique de Nice, UMR 6525 Université de Nice - Sophia Antipolis/CNRS, Parc Valrose, 06108 Nice Cedex 2, France

5 INAF-Osservatorio Astrofisico di Arcetri, Istituto Nazionale di Astrofisica, Largo E. Fermi 5, 50125 Firenze, Italy

${ }^{6}$ European Southern Observatory, Casilla 19001, Santiago 19, Chile

7 ONERA/DOTA, 29 av de la Division Leclerc, BP 72, 92322 Chatillon Cedex, France

8 Centre de Recherche Astronomique de Lyon, UMR 5574 Université Claude Bernard/CNRS, 9 avenue Charles André, 69561 Saint Genis Laval Cedex, France

9 Division Technique INSU/CNRS UPS 855, 1 place Aristide Briand, 92195 Meudon Cedex, France

10 IRCOM, UMR 6615 Université de Limoges/CNRS, 123 avenue Albert Thomas, 87060 Limoges Cedex, France

11 European Southern Observatory, Karl Schwarzschild Strasse 2, 85748 Garching, Germany

12 Kiepenheuer Institut für Sonnenphysik, Schöneckstr. 6, 79104 Freiburg, Germany

13 Dipartimento di Fisica, Università degli Studi di Milano, Via Celoria 16, 20133 Milano, Italy

14 Instituut voor Sterrenkunde, KU-Leuven, Celestijnenlaan 200D, 3001 Leuven, Belgium

15 Centro de Astrofísica da Universidade do Porto, Rua das Estrelas, 4150-762 Porto, Portugal

16 Laboratoire Astrophysique de Toulouse, UMR 5572 Université Paul Sabatier/CNRS, BP 826, 65008 Tarbes Cedex, France

17 Departamento de Astronomia, Universidad de Chile, Chile 